\title{
Estimation of expected time for the development of aids under two modes of infection
}

\author{
R. Jaisankar ${ }^{1 *}$, A. Saberunnisa ${ }^{2}$ \\ ${ }^{1}$ Department of Statistics, Bharathiar University/Coimbatore-641046,Tamil Nadu, India \\ ${ }^{2}$ Department of Statistics, Bharathiar University/Coimbatore-641046, Tamil Nadu, India \\ *Corresponding Author: r_jaisankar@ rediffmail.com, \\ Available online at: www.isroset.org \\ Accepted: 13/Aug/2018, Online: 30/Aug/2018
}

\begin{abstract}
Progression of the HIV infection toward AIDS is the significant one in the study HIV infected. It is believed that when the antigenic diversity exists in the infected individual crosses a threshold value the AIDS condition begins to occur. The successive sexual contacts with an infected partner may give rise in their antigenic diversity level. In this paper, Antigenic diversity threshold is assumed to be a random variable. Taking two modes of getting the infection, namely, heterosexual and homosexual contacts with randomly selected partners, the expected time to get AIDS is derived with its variance. Numerical illustrations are provided using simulation techniques to substantiate the results.
\end{abstract}

Keywords: Acquired Immuno Deficiency Syndrome, Human Immunodeficiency virus, Antigenic Diversity Threshold.

\section{I .INTRODUCTION}

AIDS is one of the most severecrisesthat challenge this century. Theantigenic diversity imposed by HIV is afeature to be considered because of which no successful medicine or vaccine has been found against. Antigenic diversity plays a significant role in suppressing the immune system of the HIV infected patients. Nowak and May (1991) and Stilianakis (1994) have studied the concept of the existence of antigenic diversity threshold in the immune system.It is believed that if the total antigenic diversity crosses this threshold the symptomatic conditions for AIDS begin to develop in an infected individual. The time to cross the threshold of antigenic diversity is important in the sense that it gives an idea of the probable time when an infected person begins to show the symptoms of AIDS.

HIV infection and the transmission occurnormally through one source - contacts with an infected person. However, it ispossible that a person can get the transmission of HIV throughtwo sources of infections namely heterosexual contacts and homosexual contacts. Taking into the account of this fact, in this paper, a stochastic model has been developed to estimate the expected time to cross antigenic diversity threshold assuming that the individual may get the infection through randomly selected partners.

\section{ASSUMPTIONS OF THE MODEL}

(i) Over a time period, a person is assumed to have a number of homo and heterosexual contacts with persons at random which includes HIV infected partners.

(ii) Every contact with an infected person leads to the maximization of antigenic diversity

(iii) The antigenic diversity threshold of an individual is assumed to be a random variable, follows the exponential distribution.

(v) The damages thatare the contribution to the antigenic diversity level due to the events namely heterosexual contacts and homosexual contacts are statistically independent and their correspondinginter- arrival times between successive contacts are assumed to be independent and identically distributed random variables.

(vi) Over a period of time say $(0, t)$ an infected individual is having contacts with $k$ person's at random and out of which $m$ are heterosexually infected and with $l$ person's $n$ are homosexually infected and $q$ is the probability that his random partner is being infected by HIV.

\section{NOTATIONS}

$X_{i}$-A continuous random variable denoting the amount of damage caused due to the $i^{t h}$ heterosexual contact and $X_{i}{ }^{\prime} S$ assumed to be i.i.d.

$Y_{i}$ - A continuous random variable denoting the amount of damage caused due to the $i^{\text {th }}$ homosexual contact and $Y_{i}^{\prime}$ sassumed to be i.i.d. 
$f($.$) -Probabilitydensity function of X_{i}$

$g($.$) -Probability density function of Y_{i}$

$\tau_{i}$ - Inter-arrival time between two successive heterosexual contacts which are assumed to be i.i.d random variables

$\eta_{i}$ - Inter-arrival time between two successive homosexual contacts which are assumed to be i.i.d random variables

$Z$ - A random variable representing the antigenic diversity threshold level, which is assumed to follow the exponential distribution with parameter $\mu$

$T$-A random variable representing the time to cross the Antigenic diversity threshold CADT.

$W_{k}($.$) - Cumulative distribution function of \sum_{i=0}^{m} \tau_{i}$

$V_{l}($.$) - Cumulative distribution function of \sum_{i=0}^{n} \eta_{i}$

$f^{*}($.$) -Laplace transform of f($.

$g^{*}($.$) - Laplace transform of g($.

$F_{m}($.$) -Cumulative distribution function of \tilde{X}$

$G_{n}($.$) - Cumulative distribution function of \tilde{Y}$

\section{RESULTS}

The probability of having kheterosexual contacts during the interval $(0, t)$ is,

$P(K=k \mid t)=\sum_{k=0}^{\infty}\left[W_{k}(t)-W_{k+1}(t)\right]$

The probability that out of $k$ randomly chosen heterosexual partners, $m$ are infected is

$P(M=m \mid k)=\left(\begin{array}{c}k \\ m\end{array}\right) q^{m}(1-q)^{k-m}$

Similarly,the probability of having lhomosexual contacts during the interval $(0, t)$ is,

$P(L=l \mid t)=\sum_{l=0}^{\infty}\left[V_{l}(t)-V_{l+1}(t)\right]$

The probability that out oflrandomly chosen partners, $n$ are infected of homo sexual contacts

$P(N=n \mid l)=\left(\begin{array}{l}l \\ n\end{array}\right) q^{n}(1-q)^{l-n}$

The probability that the total antigenic diversity induced by $m$ heterosexual contacts and $n$ homosexual contacts does not exceed the threshold Zis given by

$$
\begin{aligned}
P[(\tilde{X}+\tilde{Y})<Z] & =\int_{0}^{\infty} P[(\tilde{X}+\tilde{Y})<z] \mu e^{-\mu z} d z \\
= & {\left[f^{*}(\mu)\right]^{m}\left[g^{*}(\mu)\right]^{n} }
\end{aligned}
$$

Then the probability that the antigenic diversity does not cross the threshold level before the time $t$ is given by

$$
\begin{aligned}
& P(T>t)=\left[\sum_{k=0}^{\infty}\left[W_{k}(t)-W_{k+1}(t)\right] \sum_{m=0}^{k}\left(\begin{array}{c}
k \\
m
\end{array}\right) q^{m}(1-\right. \\
& \left.q)^{k-m}\left[f^{*}(\mu)\right]^{m}\right] * \\
& {\left[\sum_{l=0}^{\infty}\left[V_{l}(t)-V_{l+1}(t)\right] \sum_{n=0}^{l}\left(\begin{array}{l}
l \\
n
\end{array}\right) q^{n}(1-\right.} \\
& \left.q)^{l-n}\left[g^{*}(\mu)\right]^{n}\right]
\end{aligned}
$$

$$
\begin{aligned}
& = \\
& \begin{array}{l}
\sum_{k=0}^{\infty}\left[W_{k}(t)-W_{k+1}(t)\right](1-q(1- \\
\left.\left.f^{*}(\mu)\right)\right)^{k} \quad * \sum_{l=0}^{\infty}\left[V_{l}(t)-\right.
\end{array} \\
& \left.V_{l+1}(t)\right]\left(1-q\left(1-g^{*}(\mu)\right)\right)^{l} \\
& \text { Taking } A=\left[1-q\left(1-f^{*}(\mu)\right)\right] \text { and } B=[1- \\
& \begin{array}{c}
\left.q\left(1-g^{*}(\mu)\right)\right] \\
=\sum_{k=0}^{\infty}\left[W_{k}(t)-W_{k+1}(t)\right] A^{k} * \sum_{l=0}^{\infty}\left[V_{l}(t)-V_{l+1}(t)\right] B^{l}
\end{array} \\
& P(T>t)=\left\{1-(1-A) \sum_{k=1}^{\infty}\left[W_{k}(t)\right] A^{k-1}\right\} \\
& \quad *\left\{1-(1-B) \sum_{l=1}^{\infty}\left[V_{l}(t)\right] B^{l-1}\right\} \\
& P(T \leq t)=(1-A) \sum_{k=1}^{\infty}\left[W_{k}(t)\right] A^{k-1}+(1- \\
& \text { B) } \sum_{l=1}^{\infty}\left[V_{l}(t)\right] B^{l-1}-(1-A) \sum_{k=1}^{\infty}\left[W_{k}(t)\right] A^{k-1}(1- \\
& \text { B) } \sum_{l=1}^{\infty}\left[V_{l}(t)\right] B^{l-1}
\end{aligned}
$$

Taking Laplace Transform and differentiating the above equation with respect to $t$ we get, $\quad l^{*}(s)$

$$
\begin{gathered}
=(1-A)\left[\frac{1}{w^{*}(s)}-A\right]^{-1}+(1-B)\left[\frac{1}{v^{*}(s)}-B\right]^{-1} \\
-(1-A)(1-B)\left\{\left[\frac{1}{w^{*}(s)}-A\right]^{-1} *\left[\frac{1}{v^{*}(s)}-B\right]^{-1}\right\} \\
E(T)=-\left.\frac{d l^{*}(s)}{d s}\right|_{s=0} \\
=-\left\{(1-A)\left[\frac{1}{w^{*}(s)}-A\right]^{-2}\left[\frac{1}{w^{*}(s)^{2}}\right] w^{*^{\prime}}(\mathrm{s})+\quad(1-\right. \\
B)\left[\frac{1}{v^{*}(s)}-B\right]^{-2}\left[\frac{1}{v^{*}(s)}\right] v^{*^{\prime}}(\mathrm{s}) \\
\quad-(1-A)(1-B)\left\{\left[\frac{1}{w^{*}(s)}-A\right]^{-2}\left[\frac{1}{w^{*}(s)^{2}}\right] w^{*^{\prime}}(\mathrm{s})\right. \\
\left.*\left[\frac{1}{v^{*}(s)}-B\right]^{-2}\left[\frac{1}{v^{*}(s)^{2}}\right] v^{*^{\prime}}(\mathrm{s})\right\}
\end{gathered}
$$

If the inter arrival times $\tau_{i}$ and $\eta_{i}$ are assumed to follow the exponential distribution with parameter $\lambda_{1}$ and $\lambda_{2}$ respectively, we have

$$
\begin{aligned}
& E(T)=\frac{1}{\lambda_{1} q\left(1-f^{*}(\mu)\right)+\lambda_{2} q\left(1-g^{*}(\mu)\right)} \\
& +\frac{1}{\lambda_{1} q\left(1-f^{*}(\mu)\right) \lambda_{2} q\left(1-g^{*}(\mu)\right)} \\
& V(T)=E\left(T^{2}\right)-[E(T)]^{2} \\
& V(T) \\
& =\left[\frac{1}{\lambda_{1}^{2}\left\{q\left(1-f^{*}(\mu)\right)\right\}^{2}+\lambda_{2}^{2}\left\{q\left(1-g^{*}(\mu)\right)\right\}^{2}}\right. \\
& \left.+\frac{3}{\lambda_{1}^{2}\left\{q\left(1-f^{*}(\mu)\right)\right\}^{2} \lambda_{2}^{2}\left\{q\left(1-g^{*}(\mu)\right)\right\}^{2}}\right]
\end{aligned}
$$


Assuming that $f($.$) and g($.$) follow the exponential$ distribution with means $\beta_{1}$ and $\beta_{2}$ respectively, to examine the sample mean and variance of the time to cross the ADT, a simulation study has been carried outfor various combinations of parameters. The results are furnished in form of graphs, which are given below.

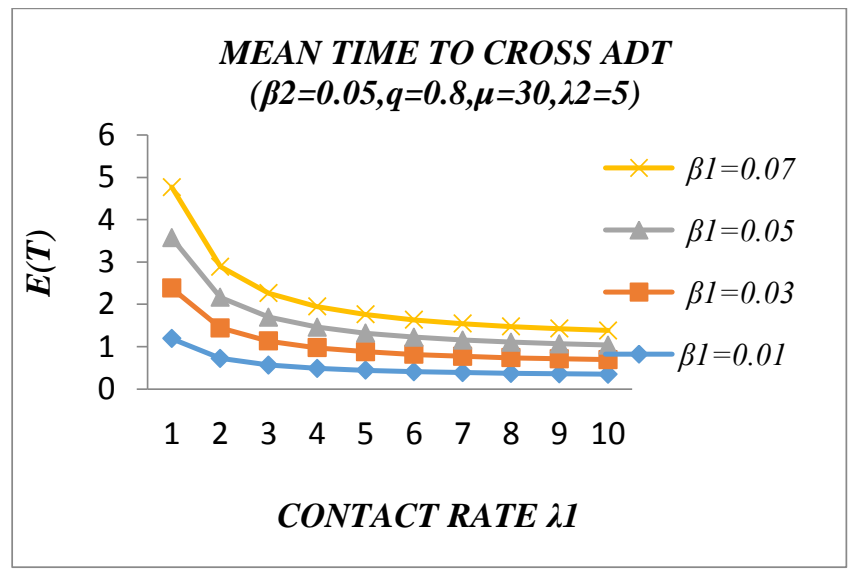

FIGURE 1.a

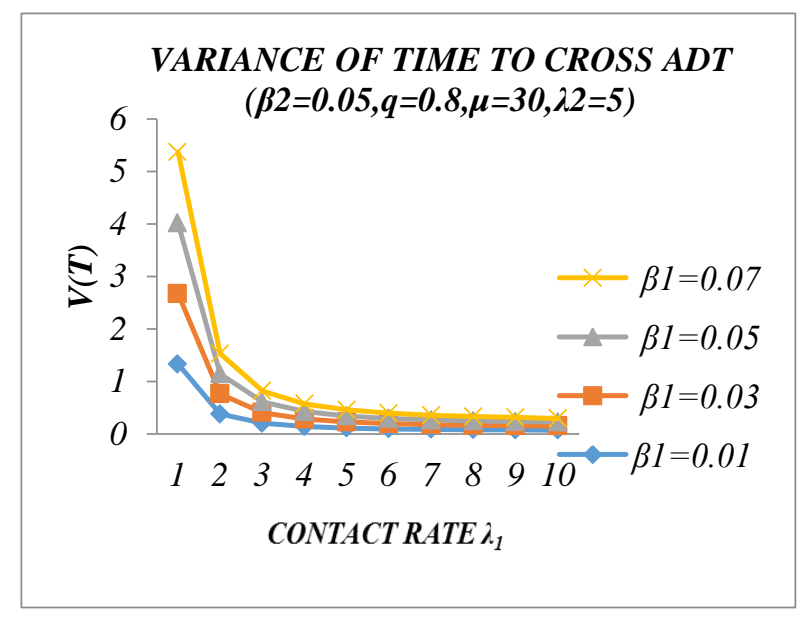

FIGURE 1.b

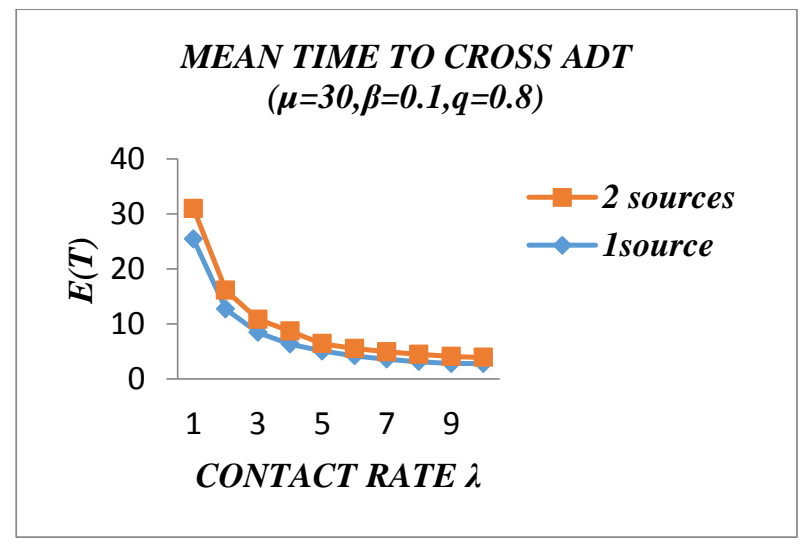

FIGURE 2.a

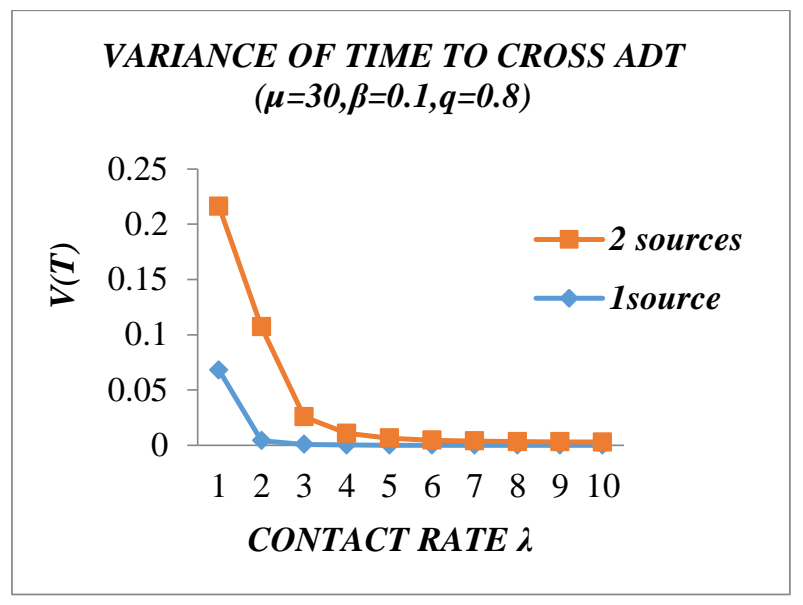

FIGURE 2.b

\section{CONCLUSION}

In fig 1.a and 1.b, the behavior of mean and variance of time to cross ADT for various values of $\beta_{1}$ have been observed for keeping $\beta_{2}, \mu, \lambda_{1}$ and $q$ fixed. As the contact rate increases both the meantime and variance to CADT decreases. But as $\beta_{1}$ increases in meantime and variance the damage caused to the immune system is moreand the same can be interpreted from the graphs that the symptoms of AIDS may be developed soon when the immune system gets more infected with viruses of higher antigenic diversity.

In figure 2.a and 2.b, a comparison is made between case having one mode of infection and two modes of infections. Here it is seen that the mean as well as the variance of time to cross the antigenic diversity threshold (ADT) are shorter in two modes of infections case when compared to one mode of infection. Therefore if the individual has two modes of sexual contacts which are more vulnerable for HIV infected subsequently he will get AIDS symptoms soon.

In all, it can be inferred that if an infected individual is exposed to more number of sexual contacts under different modes with other individuals from a group which is more vulnerable, may get the condition of AIDS soon. Hence by keeping away from additional exposure of other sources ofinfection, he can keep himself protected from getting AIDS soon.

\section{REFERENCE}

[1]. Barbara Bittner et. al."Virus Load and Antigenic Diversity".,Bulletin of Mathematical biology, Vol 59:881896,1997.

[2]. P.ChristopherLocher, VolkerHeinrichs, Doris Apt, G .Robert Whalen, "Overcoming antigenic diversity and improving 
vaccines using DNA shuffling and screening technologies", Expert Opinion on Biological Therapy, 4:589-597,2004.

[3]. R. J.De Boer, and M.C.Boerlijst,"Diversity and Virulence threshold in AIDS". Applied Mathematics. Vol.94. 544$548,1994$.

[4]. M.A .Nowak. and R.M .May, "Mathematical Biology of HIV Infection: Antigenic Variation and Diversity threshold", Mathematical Biosciences, 106, pp. 1-21, 1991.
[5]. M. A .Nowak, D. J.Stekeland, andR.M May,"Antigenic Diversity Thresholds and Hazard Functions". Mathematical Bioscience, Vol.139, 59-68, 1997.

[6]. N.I.Stillnakis, D.Schenzle and K.Dietz, "On the Antigenic diversity threshold model for AIDS", Mathematical Biosciences, 121, pp.235-247, 1994. 